\title{
Near Threshold Operation of Semiconductor Lasers and Resonant-Type Laser Amplifiers
}

\author{
Rongqing Hui, Sergio Benedetto, and Ivo Montrosset
}

\begin{abstract}
The characteristics of a semiconductor laser operating in the threshold region are investigated systematically. In this transition region from below to above threshold, the linewidth versus injection current characteristic is found to be nonmonotonic: a local minimum of linewidth just below threshold and a local maximum just above threshold are confirmed experimentally. If a semiconductor laser works below threshold as a resonant optical amplifier or optical filter, the small-signal frequency bandwidth is found to be equivalent to the spontaneous emission linewidth. When the laser amplifier is used simultaneously as a photodetector, the maximum value of photodetection sensitivity is achieved with the laser amplifier biased between 98-99\% of the threshold current. The Fokker-Planck equation method is employed in the linewidth calculation. A numerical computer simulation is also performed using the rateequation model. A reasonable agreement between theory and experiment is obtained.
\end{abstract}

\section{INTRODUCTION}

$\mathrm{I}^{\mathrm{n}}$ NTEREST in the linewidth was present from the very beginning of laser physics. Schawlow and Townes predicted that the laser line shape would be Lorentzian and that the linewidth-power product would be constant [1], this formula is, however, only valid below threshold. Above threshold, the intensity fluctuations of the laser are stabilized and this results in a $50 \%$ reduction of the linewidth-power product [2]. Semiconductor lasers differ from other kind of lasers in that they have a strong effect of phase-amplitude coupling, and this is accompanied by a $\left(1+\alpha^{2}\right)$ increasing in the linewidth. We will refer to this correction as the modified Schawlow-Townes formula for semiconductor lasers [3], where $\alpha$ is often referred to as the linewidth enhancement factor [3]-[4]. However, this modified Schawlow-Townes formula is valid only when the semiconductor laser operates above threshold. The linewidth in the transition region from below to above threshold has been calculated for gas lasers [5], [6], but not for semiconductor lasers.

Recently, it was found that single-mode semiconductor lasers, such as distributed-feedback (DFB) or distributed Bragg reflector (DBR), operating just below threshold, can be used as optical amplifiers and narrow-band optical fil-

Manuscript received June 10, 1992; revised November 24, 1992. This work was supported by CNR and Camera di Commercio di Torino under the Research Grant "All-Optical Communication Networks" and the "Progetto Finalizzato Telecommunicazioni"

The authors are with the Dipartimento di Elettronica, Politecnico di Torino, 10129 Torino, Italy.

IEEE Log Number 9209105. ters with good characteristics [7], [8]. These devices may become very important in future networks based on the optical frequency division multiple access (FDMA). Furthermore, since the electric voltage across the junction of the laser amplifier is related to the input optical signal through the depletion of the quasi-Fermi level, the device can operate simultaneously as a high-sensitivity photodetector [9], thus providing a much simplified receiver structure. If the laser operates above threshold in the presence of optical input, it can work as an injection-locked optical amplifier [10], where a different physical mechanism is involved. Therefore, a better understanding on the semiconductor laser performance in the threshold region is necessary. A detailed analysis on this subject however has not appeared so far in the literature. In this paper, we will present the results of systematic measurements on a $1.5 \mu \mathrm{m} \mathrm{InGaAsP}$ DFB semiconductor laser operating in the threshold region, such as the linewidth, the optical amplification characteristics, the frequency tuning efficiency and the photodetection sensitivity. The FokkerPlanck equation technique is employed in the linewidth calculation and a numerical computer simulation is also performed using the rate equation model. The effect of various laser parameters is evaluated.

\section{EXPERIMENT}

\section{A. Experimental Set-Up}

The experimental setup is schematically illustrated in Fig. 1. Three identical InGaAsP DFB-BH laser diodes with an emission wavelength of $1543 \mathrm{~nm}$ are used. One of them (LD1) is used, as a probe, to generate the optical signal and its output is injected into an other laser (LD2) which works near threshold as the test laser. The third DFB laser (LD3) is used as a local oscillator which beats with the output of the test laser and down-shifts the signals to radio frequency. All three lasers used in the experiment are temperature and current stabilized and in front of each of them a Faraday optical isolator is placed providing more than $70 \mathrm{~dB}$ of isolation. A PIN photodetector and a microwave spectrum analyzer are used to monitor the heterodyned IF spectrum and to evaluate the laser linewidth. A monochromator is used to see the laser mode structure and to evaluate the linewidth of the laser operating well below threshold. The spectral linewidth of the local oscillator used in the experiment is measured to 


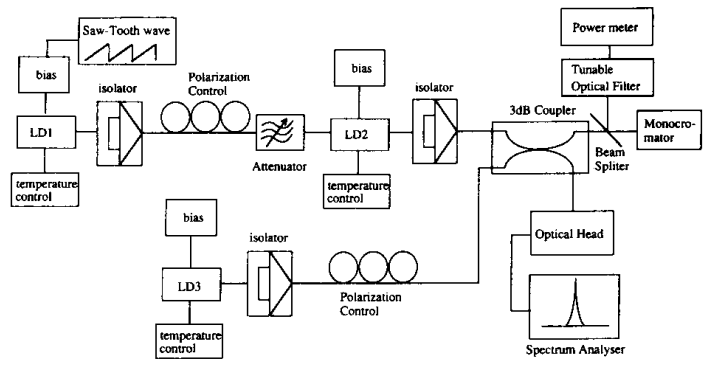

Fig. 1. Experimental setup.

be about $20 \mathrm{MHz}$ and the linewidth of the probe laser is about $60 \mathrm{MHz}$.

\section{B. Characteristics of the Solitary Laser Diode}

When the optical injection from the probe laser is blocked, the free-running characteristics of the laser amplifier can be characterized. The measured power spectrum of the laser amplifier for three values of injection current is shown in Fig. 2(a). When the laser was biased well below threshold, the spectral linewidth of the spontaneous emission is wide. As expected, increasing the bias current narrows the spectral linewidth as shown in curve b. However, this linewidth narrowing with the injection current stops when the current reaches about $98.6 \%$ of the threshold value. Further increasing the injection current results in a linewidth rebroadening. This is clearly shown in curve c. A systematic measurement of the spectral linewidth versus the normalized injection current in the transition region from below to above threshold is shown in Fig. 3. A local minimum of the linewidth below threshold and a local maximum just above threshold are evident. The linewidth-power product versus the emitted optical power is reported in Fig. 4, where the optical power has been measured at the output of the optical fiber coupled to the laser diode. Below threshold, the linewidth-power product remains constant as predicted by SchawlowTownes formula. Near threshold, this value increases gradually to reach its value given by the modified Schawlow-Townes formula. In addition, a peak appears above threshold; this is caused by the laser's relaxation oscillations: above threshold but at low power level, the frequency of the relaxation oscillation is low and cannot be distinguished from the central line, thus broadening the measured $3 \mathrm{~dB}$ linewidth. Well above threshold, the linewidth-power product increases linearly with the optical power, which indicates that there is a power-independent term in the linewidth formula. For single-mode semiconductor lasers, the residual linewidth at high optical power level has been explained by the $1 / f$ noise [11]

The central emission frequency shift versus the injection current from below to above threshold is also measured and reported in Fig. 5 for four DFB laser diodes of the same kind. This gives the information of the static frequency tuning efficiency in conventional DFB semiconductor laser amplifiers. Well below threshold, this fre-

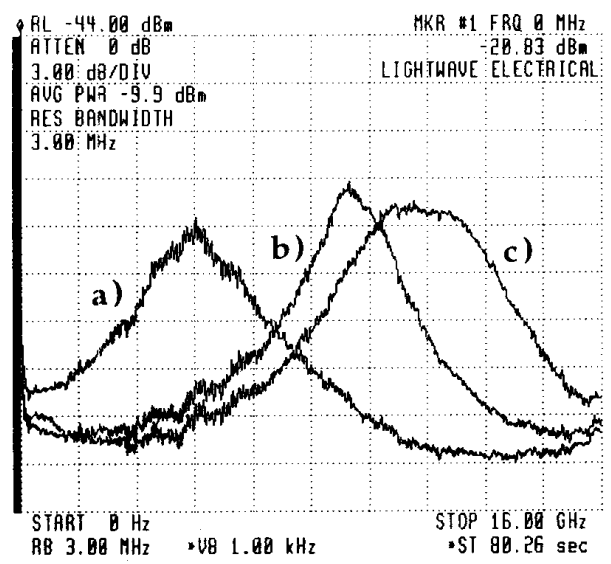

(a)

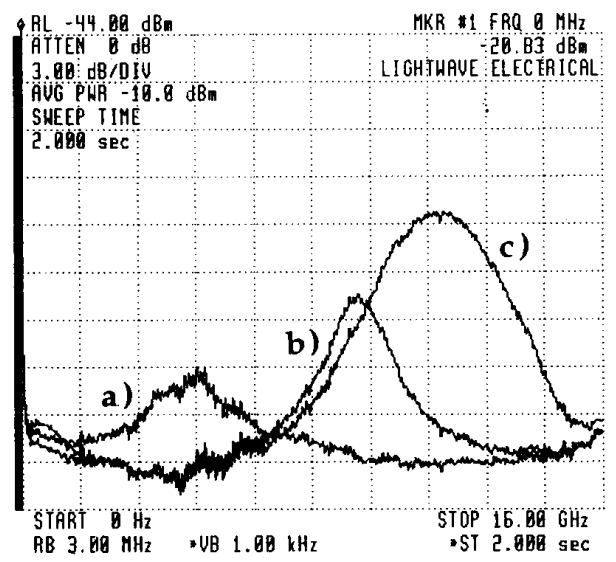

(b)

Fig. 2. (a) Measured spontaneous emission speatra with the laser amplifier biased at a) 95.2 , b) 98 , and c) $101.3 \%$. (b) Optical transmission response of the laser amplifier when the input optical signal is frequency swept. Other parameters are the same as in (a).

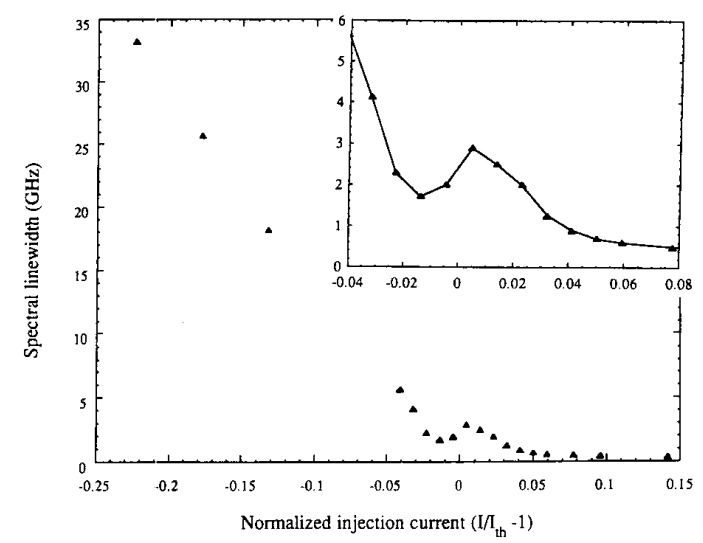

Fig. 3. Measured spectral linewidth of the laser from below to above threshold. The insert shows the part in the threshold region.

quency shift versus current is originated from the carrier and thermal effects: carrier population increases linearly with the injection current resulting in the blue shift of the 


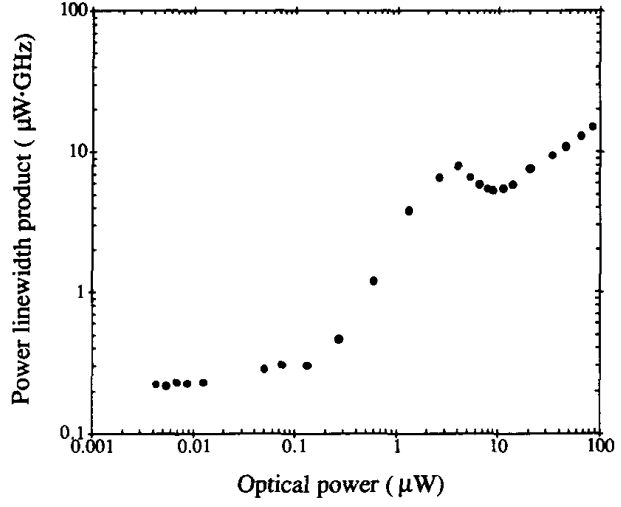

Fig. 4. The measured linewidth-power product versus the optical power from below to above threshold. Where the optical power has been measured at the output of the optical fiber which was coupled to the laser.

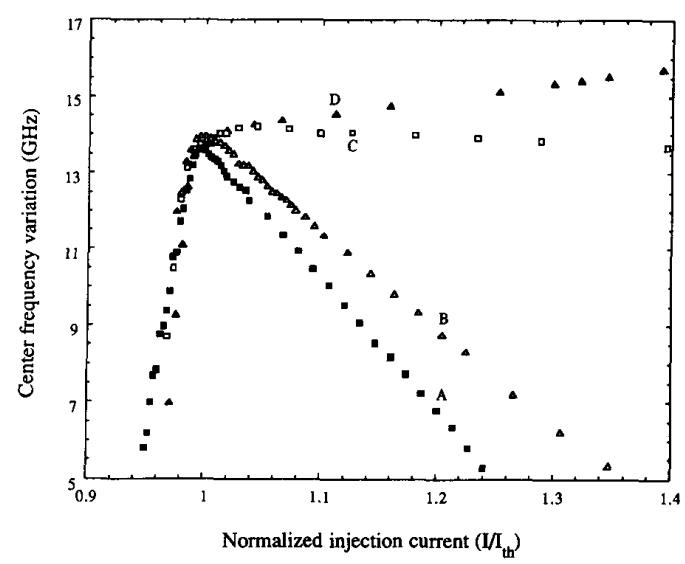

Fig. 5. The lasers' center frequency shift versus the normalized injection current for four InGaAsP DFB-BH lasers with the same structure.

wavelength, whereas the actual temperature of the laser's active region increases with the injection current but its effect of red shift is less important. Near threshold, however, the carrier density begins to be clamped to its threshold value and the wavelength tuning efficiency versus injection current is gradually reduced. Well above threshold, the static wavelength shift versus injection current is mainly determined by the thermal effect and the nonlinear gain suppression. If the thermal effect is more important than the effect of nonlinear gain suppression, wavelength versus current characteristic changes its sign from below to above threshold (e.g., laser A and B), oth erwise the blue shift of the wavelength is monotonic (e.g. laser $\mathrm{C}$ and $\mathrm{D}$ ) as shown in Fig. 5. This information may be useful in evaluating the carrier effect below threshold, the thermal effect just above threshold and the nonlinear gain saturation well above threshold. From Fig. 5, it also appears that the absolute values of the slope of the curves above threshold are much less than below threshold (more than five times). Since the thermal effect does not change very much from below to above threshold, this implies that in conventional DFB-BH semiconductor lasers, as we used in the experiment, the carrier effect is predominant in determining the static wavelength shift below threshold. This result support the linewidth enhancement factor measurement method proposed by Kikuchi [12]. In this method, $\alpha$ is expressed as $\alpha=-2\left(\Delta f_{0}\right) /[\Delta(\delta \nu)]$, where $\Delta f_{0}$ and $\Delta(\delta \nu)$ are, respectively, the center frequency shift and the changes of the linewidth induced by an increment of the injection current through the change of the carrier population. Comparing Fig. 5 (laser D) with Fig. 3, the $\alpha$ value of this laser is estimated to be about 5 .

\section{Characteristics of Optical Amplification}

By injecting the optical signal from the probe laser into the laser amplifier, the optical gain and its bandwidth can be evaluated. In order to have a frequency swept optical signal, a low frequency $(100 \mathrm{~Hz})$ direct saw-tooth current modulation is applied to the probe laser. The modulation index is chosen such that the signal wavelength is swept over the frequency range of the laser amplifier's resonance (about $16 \mathrm{GHz}$ in our case) while the intensity modulation is negligible. With this swept optical signal injection, the output optical spectrum from the laser amplifier is shown in Fig. 2(b). In obtaining this figure, the sweep time of the spectrum analyzer was set to be much longer than the sweep time of the probe laser. Each curve in Fig. 2 (b) is obtained with the laser amplifier operating in the same condition as in Fig. 2(a). The property of optical response of the laser amplifier can be evaluated by comparing Fig. 2(b) with (a). In obtaining Fig. 2(b), the injected optical power was kept at about $-45 \mathrm{dBm}$. It is interesting to note, from Fig. 2, that below threshold, the bandwidth of small-signal optical amplification is essentially equivalent to the spontaneous emission linewidth, while just above threshold, the line shape of the optical amplification is distorted compared with the spontaneous emission line shape and the distortion is dependent on the amount of optical injection, even when this is very small.

Since the electric voltage across the junction of the laser amplifier is related to the carrier population inside its active cavity through the quasi-Fermi level of the gain medium, the static properties of the previously demonstrated laser amplifier photodetector [9] can be investigated by measuring the junction electric voltage variation as a function of the input optical signal. Fig. 6 reports the measured junction electric voltage variation versus signal light frequency detuning for four different optical injection levels with the laser amplifier biased at $98 \% I_{t h}$. Asymmetrical amplification line shape and bistability have also been found when the injected optical power becomes higher. This figure is similar to that reported in [13], where optical outputs were detected. Since the photodetection effect is closely dependent on the bias level of the laser amplifier, we also measured this photodetection characteristic versus frequency detuning for several different bias levels with the optical injection level kept constant, and this is reported in Fig. 7. Surprisingly, the photodetection found its maximum sensitivity with the laser amplifier biased at about $98 \%$ of the threshold. Further 


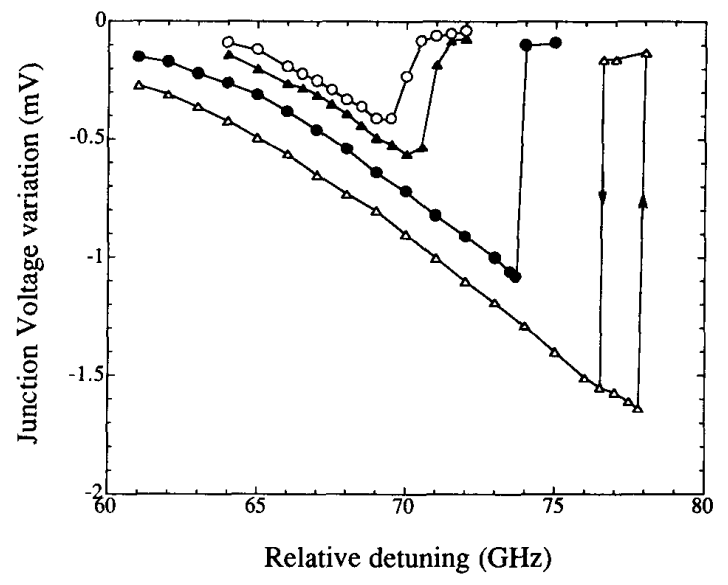

Fig. 6. Measured junction electric voltage of the laser amplifier versus the frequency detuning of the injected signal light for four different injection optical power: $P=-40 \mathrm{dBm}(00),-36.5 \mathrm{dBm}(\Delta),-31 \mathrm{dBm}(\bullet)$, and $-27 \mathrm{dBm}(\triangle)$. The laser amplifier was biased at about $96.5 \%$ of the threshold current.

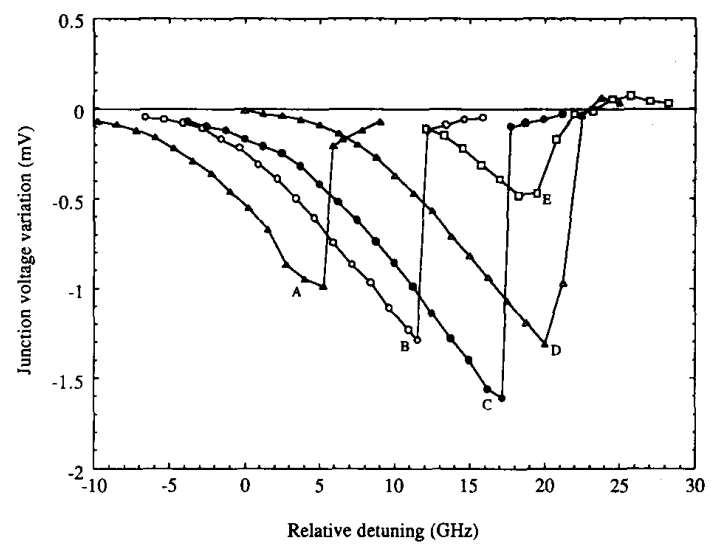

Fig. 7. Junction electric voltage of a laser amplifier versus the frequency detuning of the signal light for five different bias levels of the laser amplifier: $I=92.6 \% I_{t h}(\mathrm{~A}), 95.2 \% I_{t h}(\mathrm{~B}), 97.9 \% I_{t h}(\mathrm{C}), 100 \% I_{t h}(\mathrm{D})$, and $101.9 \% I_{t h}$. The injected optical power was kept at about $-33 \mathrm{dBm}$.

increasing the bias level reduces the photodetection sensitivity and finally, the laser amplifier is partially injection locked, which is indicated by the fact that the junction voltage variation has a positive value as shown in curves $D$ and $E$ of Fig. 7. A systematic measurement of the laser amplifier photodetection sensitivity versus the bias level is reported in Fig. 8 for two different values of optical input power. In this measurement, for each bias level, a proper value of frequency detuning was chosen which corresponds to the maximum junction voltage signal. Since both the strong nonlinear effect and the decreasing in the photodetection sensitivity will arise when a laser amplifier is biased very close to its threshold, system performances degradation will happen if the laser amplifier optical filter/photodetector is biased too close to the threshold. This has been verified in our previous transmission system experiment reported in [14].

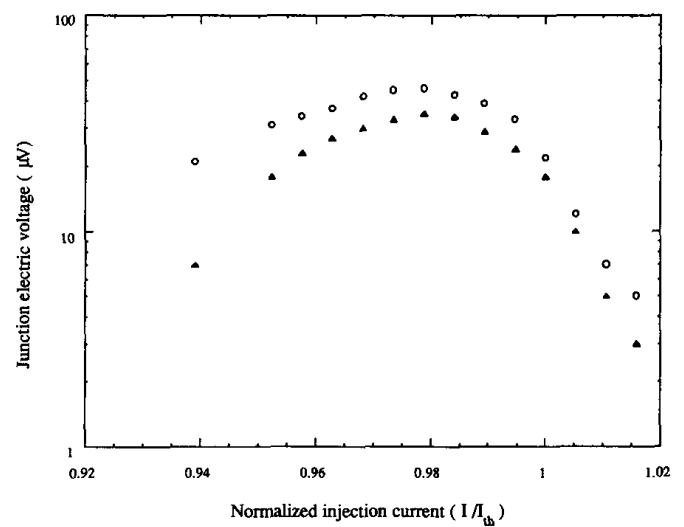

Fig. 8. Measured maximum junction voltage signal versus the normalized bias level of the laser amplifier for two different optical injection powers: $P_{i}=-48 \mathrm{dBm}$ (triangles) and $P_{i}=-43 \mathrm{dBm}$ (circles).

\section{Semiconductor Laser Linewidth Near THRESHOLD}

The linewidth of semiconductor lasers has been studied extensively in the last decade. If a semiconductor laser operates below threshold, its linewidth is determined by the Schawlow-Townes formula [1]. On the other hand, if a semiconductor laser operates above threshold, the linewidth follows the modified Schawlow-Townes formula in which the linewidth enhancement factor plays an important role [3], [4]. Since the phase-amplitude coupling effect is very strong in semiconductor lasers, its linewidth change through the threshold region is qualitatively different from that of gas lasers where the phase-amplitude coupling is negligible [5].

It is commonly accepted that the performance of a semiconductor laser can be well described by a set of Langevin rate equations for the complex field $E(t)$ and the carrier population $N(t)$, respectively, [4]:

$$
\begin{aligned}
& d E(t) / d t=\frac{\Gamma G(N)-1 / \tau_{p}}{2}(1-i \alpha) E(t)+F_{E}(t) \\
& d N(t) / d t=I / q v-N / \tau_{e}-G(N) P+F_{N}(t)
\end{aligned}
$$

where $G(N)=G_{N}\left(N-N_{0}\right)$ is the material gain, $G_{N}$ is the differential gain coefficient, $\Gamma$ is the confinement factor, $N_{0}$ is the carrier population at transparency, $\tau_{p}$ is the photon lifetime, $\alpha$ is the linewidth enhancement factor, $I$ is the injection current, $P=|E|^{2}$ is normalized to be equal to the photon population, $v$ is the cavity volume, $\tau_{e}$ is the effective carrier lifetime, and $q$ is the electron charge. $F_{E}(t)$ and $F_{N}(t)$ are the Langevin forces for the field and the carrier number, the latter can usually be omitted because the shot noise contribution to the linewidth is negligible [4]. The Langevin forces for the field satisfies the correlation relation [4]:

$$
\left\langle F_{E}(t) F_{E}^{*}(u)\right\rangle=R \delta(t-u)
$$

where $\delta$ represents a delta function, $R=B \cdot B_{s p} N^{2} / v$ is the spontaneous emission rate, $B$ is the radiative recom- 
bination rate, and $B_{s p}$ is the spontaneous emission factor indicating the fraction of the spontaneously emitted photons that coupled into the laser mode. In the mean-field approximation used in (1) and (2), the nonlinear gain and the spatial-hole-burning effects have been omitted. This approximation is valid because in the low-power condition these contributions are negligible. Since in this paper we consider the linewidth, which is related only to the low frequency part of the field fluctuation, we can use the adiabatic approximation of the carrier population. This implies that the carrier population variation adiabatically follows the instantaneous variation of the photon population. With this approximation, (2) reduces to the simple form:

$$
G \approx G_{0}\left(1-P / P_{s}\right)
$$

where $G_{0}=G_{N}\left[I \tau_{e} /(q v)-N_{0}\right]$ is the small-signal gain, $P_{s}=\left(\Gamma G_{N} \tau_{e}\right)^{-1}$ is the saturation photon number and in (4) $P \ll P_{s}$ is assumed because in this analysis we are interested only in the laser behavior in the threshold region. The Fokker-Planck equation corresponding to (1) and (4) is [15]:

$$
\begin{aligned}
\frac{\partial \odot}{\partial t}= & -\frac{\partial}{\partial E}\left\{(1-i \alpha)\left[\frac{G_{0}-1 / \tau_{p}}{2}-\frac{G_{0}|E|^{2}}{2 P_{s}}\right] E \mathcal{P}\right\} \\
& -\frac{\partial}{\partial E^{*}}\left\{(1+i \alpha)\left[\frac{G_{0}-1 / \tau_{p}}{2}-\frac{G_{0}|E|^{2}}{2 P_{s}}\right]\right. \\
& \left.\cdot E^{*} \odot\right\}+R \frac{\partial^{2} \odot}{\partial E \partial E^{*}}
\end{aligned}
$$

Where $\mathcal{P}=\mathcal{P}\left(E, t \mid E_{0}, t_{0}\right)$ is a conditional probability. Usually we can write the complex electric field in the polar representation $E=r e^{i \phi}$, where $r=\sqrt{P}$ is the field amplitude, and $\phi$ is the phase. In this representation, the Fokker-Planck equation becomes:

$$
\begin{aligned}
\frac{\partial \odot}{\partial t}= & \frac{R}{4} \frac{\partial^{2} \odot}{\partial r^{2}}-\left(\frac{G_{0}-1 / \tau_{p}}{2}-\frac{G_{0} r^{3}}{2 P_{s}}-\frac{R}{4 r}\right) \frac{\partial \odot}{\partial r} \\
& -\left(G_{0}-\tau_{p}-\frac{2 G_{0}}{P_{s}} r^{2}\right) \odot-\alpha\left(\frac{G_{0}-\tau_{p}}{2}\right. \\
& \left.-\frac{G_{0}}{2 P_{s}} r^{2}\right) \frac{\partial \odot}{\partial \phi}+\frac{R}{4 r^{2}} \frac{\partial^{2} \odot}{\partial \phi^{2}} .
\end{aligned}
$$

In order to eliminate the phase variable $\phi$ and to simplify the equation, the following transformations on $\mathcal{P}, r$ and $t$ are used:

$$
\begin{aligned}
\mathcal{U} & =\int \rho e^{i \phi} d \phi \\
\hat{r} & =\left(\frac{2 G_{0}}{R P_{s}}\right)^{1 / 4} r
\end{aligned}
$$

and

$$
\hat{t}=\left(\frac{R G_{0}}{8 P_{s}}\right)^{1 / 2} t
$$

The Fokker-Planck equation is then reduced to:

$$
\begin{aligned}
\frac{\partial \mathcal{U}}{\partial \hat{t}}= & \frac{\partial^{2} \mathcal{U}}{\partial \hat{r}^{2}}-\left(x \hat{r}-\hat{r}^{3}-\hat{r}^{-1}\right) \frac{\partial \mathcal{U}}{\partial \hat{r}} \\
& +\left[4 \hat{r}^{2}-2 x-\hat{r}^{-2}+i \alpha\left(x-\hat{r}^{2}\right)\right] \mathcal{U}
\end{aligned}
$$

where $x=\left(G_{0}-\tau_{p}\right) \sqrt{2 P_{s} /\left(R G_{0}\right)}$.

A Sterm-Liuvell transformation

$$
\mathcal{u}=\frac{\vartheta}{\sqrt{\hat{r}}} \exp \left(\frac{1}{8} \hat{r}^{4}+\frac{x}{4} \hat{r}^{2}\right)
$$

eliminates the term with the first-order derivative to $\hat{r}$ and reduces the Fokker-Planck equation to the following form:

$$
\begin{aligned}
\frac{\partial \vartheta}{\partial \hat{t}}= & \frac{\partial^{2} \vartheta}{\partial \hat{r}^{2}}+\left\{-\frac{1}{4} \hat{r}^{6}+\frac{x}{2} \hat{r}^{4}+\left(2-\frac{x^{2}}{4}\right) \hat{r}^{2}-x\right. \\
& \left.-\frac{3}{4 \hat{r}^{2}}-i \alpha\left(\hat{r}^{2}-x\right)\right\} \vartheta .
\end{aligned}
$$

Suppose now that the power spectrum of the complex field is nearly Lorentzian, the correlation function $\langle E(\tau-$ t) $\left.E^{*}(\tau)\right\rangle$ must be dominated by the lowest nonzero eigenvalues of the following non-Hermitian complex eigenequation:

$$
\begin{gathered}
\frac{\partial^{2} \vartheta_{n}}{\partial \hat{r}^{2}}+\left\{-\frac{1}{4} \hat{r}^{6}+\frac{x}{2} \hat{r}^{4}+\left(2-\frac{x^{2}}{4}\right) \hat{r}^{2}-x-\frac{3}{4 \hat{r}^{2}}\right. \\
\left.-i \alpha\left(\hat{r}^{2}-x\right)\right\} \nabla_{n}=-\lambda_{n} \nabla_{n}
\end{gathered}
$$

where $\lambda_{n}$ is the complex eigenvalue. Equation (13) can be easily solved using a simple numerical approach and the complex eigenvalue with the lowest nonzero real part $\lambda_{0}$ can be obtained. The correlation function of the complex field can then be expressed as:

$$
\left\langle E(\tau-t) E^{*}(\tau)\right\rangle=\langle P\rangle \exp \left[-\left(\frac{R G_{0}}{8 P_{s}}\right)^{1 / 2} \lambda_{0} t\right] .
$$

The linewidth of the power spectra is determined by

$$
\Delta \nu=\left(\frac{R G_{0}}{8 P_{s}}\right)^{1 / 2} \operatorname{Re}\left(\lambda_{0}\right) / \pi
$$

where $\operatorname{Re}\left(\lambda_{0}\right)$ represents the real part of $\lambda_{0}$, while the imaginary part of $\lambda_{0}$, represented by $\operatorname{Im}\left(\lambda_{0}\right)$, determines the relative shift of the central frequency:

$$
\Delta f_{0}=\left(\frac{R G_{0}}{8 P_{s}}\right)^{1 / 2} \operatorname{Im}\left(\lambda_{0}\right) / \pi \text {. }
$$

In the linewidth calculation presented above, only the lowest order eigenvalue was included. This implies that the laser's line shape has been considered to be Lorentzian. The calculated linewidth versus injection current characteristics are shown in Fig. 9. The linewidths pre- 


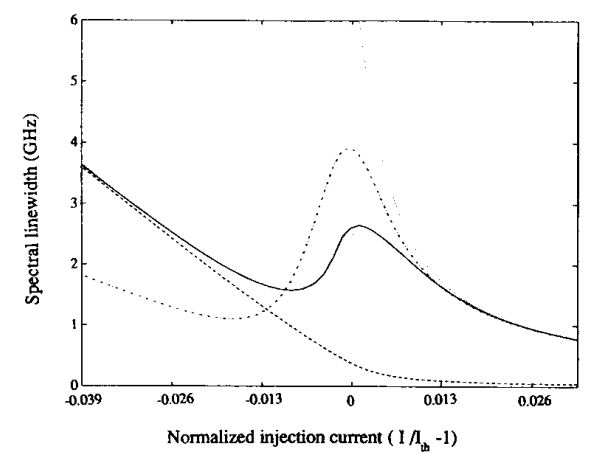

Fig. 9. Calculated spectral linewidth versus the normalized injection current with $\alpha=6$ and $B_{s p}=2.8 \times 10^{-5}$. The solid line is the result obtained by the Fokker-Planck equation method. The dash-dotted line is obtained from the linearized rate equation method. The dashed line and the dotted line indicate the linewidths predicted by the Schawlow-Townes formula and the modified Schawlow-Townes formula, respectively. The threshold current is $15.4 \mathrm{~mA}$.

dicted by the Schawlow-Townes formula [1]:

$$
\Delta \nu=R /(2 \pi P)
$$

for below threshold operation and by the modified Schawlow-Townes formula [4]:

$$
\Delta \nu=R\left(1+\alpha^{2}\right) /(4 \pi P)
$$

for above threshold operation are also represented in the same figure. The average optical power $P$ used in this figure is numerically computed using the stationary parts of (1) and (4). The parameters used in the calculation to obtain Fig. 9 are: $\alpha=6, B=10^{-16} \mathrm{~m}^{3} \mathrm{~s}^{-1}, v=10^{-16} \mathrm{~m}^{3}$, $B_{s p}=2.8 \times 10^{-5}, G_{N}=5.6 \times 10^{3} \mathrm{~s}^{-1}, N_{0}=10^{8}$, and $\alpha_{0}=6.372 \times 10^{11} \mathrm{~s}^{-1}$. The threshold current in Fig. 9 is $I_{t h}=15.4 \mathrm{~mA}$. In the region well below threshold, where the phase-amplitude coupling is negligible, the linewidth decreases with the injection current in accordings with the Schawlow-Townes formula [1], [2]. As the threshold is approached, the material gain is more and more restricted to its threshold value and the phase-amplitude coupling becomes more and more important. The linewidth changes gradually from what is predicted by the Schawlow-Townes formula to the modified SchawlowTownes formula [4]. In Fig. 9, a local minimum of the linewidth is achieved at the current $I \approx 0.991 I_{t h}$ and then a local maximum is reached with the injection current just above the operating threshold. Since, as we have already demonstrated in the experiment, the small-signal optical bandwidth is equivalent to the spontaneous emission linewidth below threshold, this local minimum of the linewidth sets a limitation on the minimum optical bandwidth achievable in the resonant-type semiconductor laser amplifiers. This result is in contrast to the conventional concept that a narrower small-signal optical bandwidth can be obtained by indefinitely approaching the threshold point [7], [8]. The linewidth versus current characteristic can also be evaluated by linearizing the rate equation (1)-(2) and calculating the frequency noise spectrum at zero frequency [16], [17]. An analytical expression of the line-

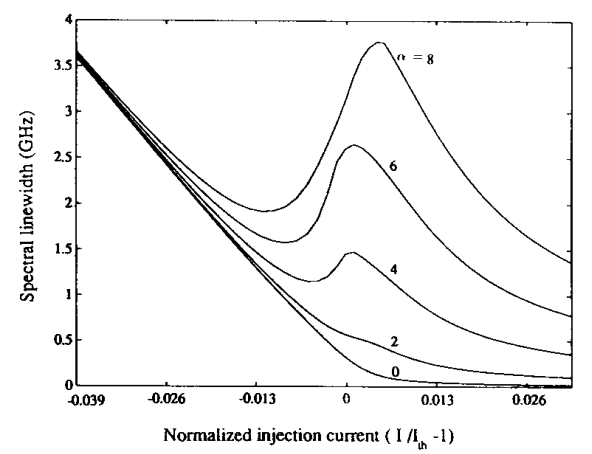

Fig. 10. Calculated linewidth for the values of the linewidth enhancement factor reported in the figure. Other parameters are the same as those in Fig. 9.

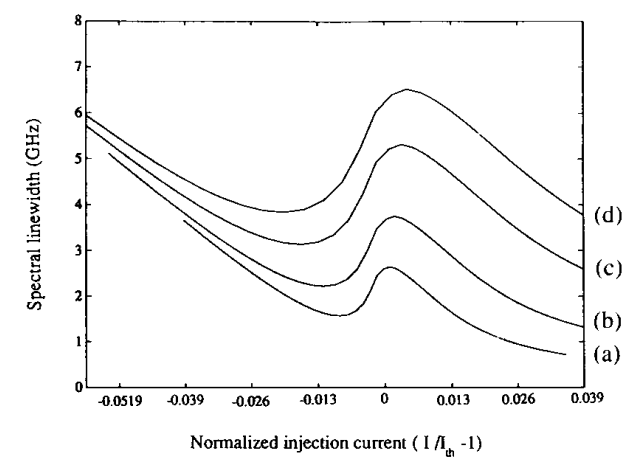

Fig. 11. Calculated linewidth for different values of spontaneous emission factor $B_{s p}=$ (a) $2.8 \times 10^{-5}$, (b) $5.6 \times 10^{-5}$, (c) $1.12 \times 10^{-4}$. and (d) $1.68 \times 10^{-4}$. Other parameters are the same as those in Fig. 9.

width can be obtained in this way if the intensity is considered to be constant. However, since the intensity fluctuation is omitted in this linewidth calculation, the predicted linewidth is $3 \mathrm{~dB}$ lower than that predicted by the Schawlow-Townes formula when the laser operates below threshold. It is also noted that just above threshold, this analytical calculation overestimates the linewidth. The reason comes also from the assumption of the stabilized intensity, as all the instabilities were coupled to the phase fluctuations, which enhances the linewidth in a much stronger way. As a comparison, the linewidth calculated using the linearized rate equation approximation is also plotted in Fig. 9 in a dash-dotted line. Figs. 10 and 11 show, respectively, the effect of the linewidth enhancement factor $\alpha$ and the spontaneous emission factor $\boldsymbol{B}_{s p}$. Increases in both $\alpha$ and $\boldsymbol{B}_{s p}$ will enlarge the transition region and increase the minimum optical bandwidth achievable in a semiconductor laser amplifier. This means that a semiconductor laser with low linewidth enhancement factor and low spontaneous emission factor is preferable in narrowband optical filter applications.

The knowledge of laser linewidth versus emitted optical power below and above threshold has recently been used to evaluate the linewidth enhancement factor $\alpha$ [18]. This can be easily understood by a comparison between 


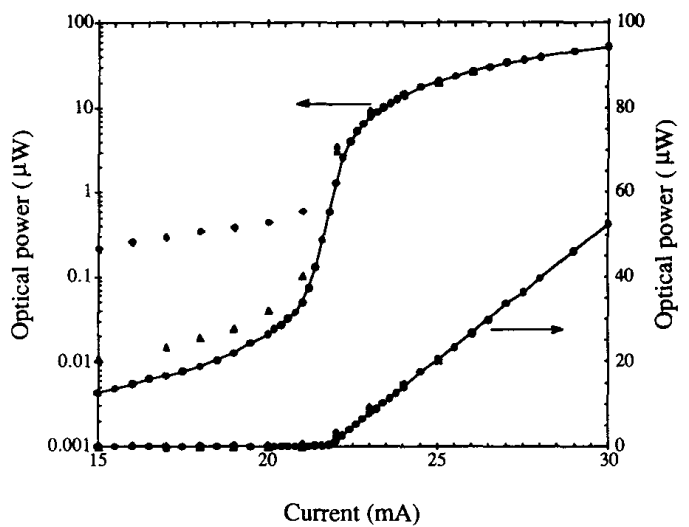

Fig. 12. Measured optical power versus injection current with different bandwidth of the optical filter: $1 \mathrm{~nm}$ (circles), $5 \mathrm{~nm}$ (triangles), and $\infty$ (rectangles).

the Schawlow-Townes formula below threshold (17) and the modified Schawlow-Townes formula above threshold (18). Obviously in this method, the resulted $\alpha$ value is closely dependent on how the optical power is measured. Since both the Schawlow-Townes formula and its modified form are valid only in the single-mode case, the optical power used in this $\alpha$ measurement would have to be the single-mode power. Usually, if a DFB laser operates above threshold, this single-mode condition can be guaranteed by its high side-mode suppression ratio. However, in the case of below threshold operation, side longitudinal modes may raise gradually, except for some specially designed laser structure where sidelobe suppression could be kept high even below threshold. We have measured the $P$-I relationship of a conventional DFB-BH semiconductor laser, whose output was passing through an adjustable optical filter. When the filter's bandwidth is infinite, so that all the powers from side modes and the spontaneous emission outside the stop-band are included, the result is shown by rectangles in Fig. 12. With the filter bandwidth of $5 \mathrm{~nm}$, the $P-I$ curve is indicated by the triangles in Fig. 12. In this case only two sidelobes were included. Solid circles in the same figure indicate the measured $P-I$ curve for the filter bandwidth of $1 \mathrm{~nm}$ where only the main mode is included. In linear scale, the three curves are indistinguishable. However, a great difference is evident below threshold when those curves are plotted in a logarithmic scale. Obviously, only the values measured in the last case are relevant in evaluating the $\alpha$ value using the abovementioned method. In our case, the $\alpha$ value is measured to be about 5 with this method, which agrees with the result of the other method described in Section II.

\section{COMPuter Simulation}

Previous static [7], [19], [20] and determinestic [8] studies predicted many characteristics of resonant-type semiconductor laser amplifiers. From these studies it seems that the small-signal optical bandwidth could be infinitely narrow and the optical gain could be infinitely large when the injection current of the laser amplifier approaches the threshold value [7], [8]. However this is not the case since we have experimentally observed not only the gain saturation but also the gain reduction very near or just above threshold. In order to give a systematic analysis of laser amplifiers operating near threshold, it is useful to include the random spontaneous emission noise and partially injection locking effect into the theoretical model and to see to what extent the rate equation model can explain the experimental observations in resonant-type semiconductor laser amplifiers. Since these effects are difficult to be described in an analytical form, we give a dynamic numerical simulation in this section.

The computer simulations are based on two sets of rate equations more general than (1)-(2). The rate equations for the laser amplifier and the signal lasers are as follows [21]:

$$
\begin{aligned}
\frac{d P}{d t}= & {\left[\Gamma G(N, P)-1 / \tau_{p}\right] P+\frac{1}{\tau_{i}} } \\
& \cdot \operatorname{Re}\left\{\left(\kappa P_{i} P\right)^{1 / 2} \exp [-i(\Omega t-\psi)]\right\}+R+F_{P}(t) \\
\frac{d \phi}{d t}= & \frac{\alpha}{2}\left[\Gamma G(N, P)-1 / \tau_{p}\right]+\frac{1}{2 \tau_{i}} \\
& \cdot \operatorname{Im}\left\{\left(\kappa P_{i} P\right)^{1 / 2} \exp [-i(\Omega t-\psi)]\right\}+F_{\phi}(t) \\
\frac{d N}{d t}= & I / q v-N / \tau_{s}-G(N, P) P+F_{N}(t) \\
\frac{d P_{i}}{d t}= & {\left[\Gamma_{i} G_{i}\left(N_{i}, P_{i}\right)-1 / \tau_{p_{i}}\right] P_{i}+R_{i}+F_{P_{i}}(t) } \\
\frac{d \phi_{i}}{d t}= & \frac{\alpha_{i}}{2}\left[\Gamma_{i} G_{i}\left(N_{i}, P_{i}\right)-1 / \tau_{p_{i}}\right]+F_{\phi_{i}}(t) \\
\frac{d N_{i}}{d t}= & I_{i} / q v-N_{i} / \tau_{s_{i}}-G_{i}\left(N_{i}, P_{i}\right) P_{i}+F_{N_{i}}(t)
\end{aligned}
$$

Equations (19) to (24) are the rate equations of the intensity, phase, and carrier population for the laser amplifier and the signal laser, respectively. The subscript $i$ indicates parameters of the signal laser. $\kappa$ represents the coupling coefficient between the probe and the laser amplifier, $\psi=\phi_{i}-\phi$ is the relative phase detuning. $1 / \tau_{s}=$ $1 / \tau_{s 1}+\left(1 / \tau_{s 2}\right) N+\left(1 / \tau_{s 3}\right) N^{2}$, where $1 / \tau_{s 1}=10^{9} \mathrm{~s}^{-1}$, $1 / \tau_{s 2}=10^{-16} \mathrm{~s}^{-1} \mathrm{~m}^{3}$, and $1 / \tau_{s 3}=3 \times 10^{-29} \mathrm{~s}^{-1} \mathrm{~m}^{6}$ are the nonradiative, radiative, and Auger recombination coefficient, respectively. Other parameters are the same as defined in the last section except for a subscript $i$ is used to indicate the parameters of the probe laser. Langevin forces $F_{p}(t), F_{\phi}(t)$, and $F_{N}(t)$, represent the fluctuations in intensity, phase, and carrier population, respectively, satisfy the usual correlation relations [4]:

$$
\left\langle F_{p}(t) F_{q}(u)\right\rangle=2 D_{p q} \delta(t-u)
$$

where the diffusion coefficients are given by: $2 D_{P P}=$ $2 R P ; 2 D_{\phi \phi}=R / 2 P ; 2 D_{P \phi}=0 ; 2 D_{P N}=-2 R P ; 2 D_{N \phi}=$ 
0 , and $2 D_{N N}=2\left(R P+N / \tau_{s}\right)$. Similar expressions for the diffusion coefficients can be written for the probe laser by simply adding a subscript $i$ to each parameter.

Equations (19) to (24) are solved numerically, in the time domain, by using a fourth-order Runge-Kutta algorithm. The Langevin noises are simulated by independent Gaussian random noise generators with the amplitude given by (25). The optical spectrum are evaluated through the fast Fourier transformation technique. The computer simulation package TOPSIM [22] is used in the simulation process. When the coupling coefficient $\kappa$ is zero, the rate equations can be solved separately for the laser amplifier and the probe laser. The optical power spectrum, the linewidth and the carrier population for a solitary laser diode can be obtained in this way. Three typical power spectrum are shown in Fig. 13, which are comparable with the experimentally measured results in Fig. 2(a). The calculated linewidth-power product from below to above threshold is reported in Fig. 14. As a comparison, the linewidth-power product calculated by the Fokker-Planck equation method is also reported in the same figure. Good agreement is obtained except in the region just above threshold. In this region, as has been explained in the experimental part, the spectral lineshape is not exactly Lorentzian, two relaxation oscillation peaks at low frequency are not distinguishable from the main peak, thus making the simulated $3 \mathrm{~dB}$ linewidth larger than that predicted by the Fokker-Planck equation technique, where only the lowest order eigenvalue was included. On the other hand, the simulated results agree well with the experimental ones reported in Fig. 4. Similar computer simulations have been carried out by Kikuchi [23]. Since where a value $\alpha=2$ was used, the linewidth rebroadening near threshold was not predicted.

With the optical signal injected, from the probe laser, into the laser amplifier, the effect of optical amplification can be observed. Fig. 13 also shows the peak height of the amplified optical signal with different frequency detuning by open circles while the injected optical power was kept at a constant value of $-50 \mathrm{dBm}$. This figure agrees well with the experimentally measured results reported in Fig. 2(b). In order to verify the measured junction electric voltage response to the injected optical signal as reported in Fig. 8, the carrier population variation caused by the injected optical signal has been obtained by simulation. Since the junction electric voltage is linearly related to the carrier population through the variation of the quasi-Fermi level [24], the calculated carrier density variation reflects qualitatively the junction voltage signal. The calculated results are given in Fig. 15 for two different spontaneous emission factor $\boldsymbol{B}_{s p}$. Fig. 15 agrees qualitatively with the experimentally measured result in Fig. 8. It has previously been thought that below threshold, optical amplification would increase with the bias level till the threshold point [10], [25]. Our experimental and simulated results reveal however that the maximum photodetection sensitivity is obtained a little below threshold. The physical explanation is that even below threshold (but

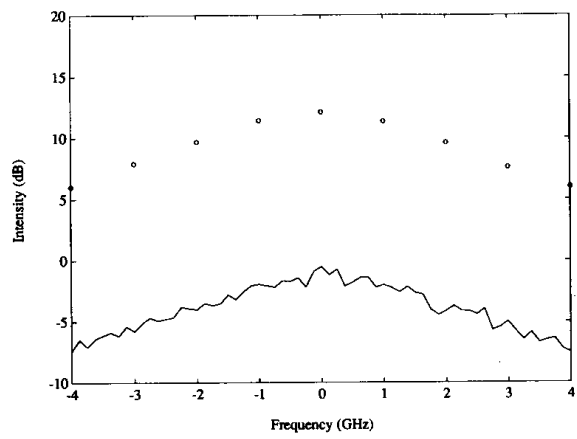

(a)

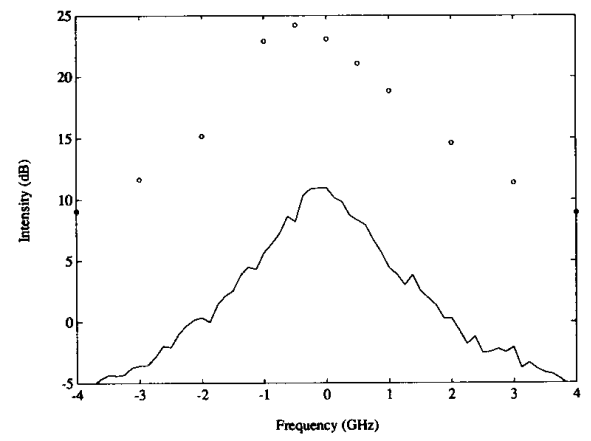

(b)

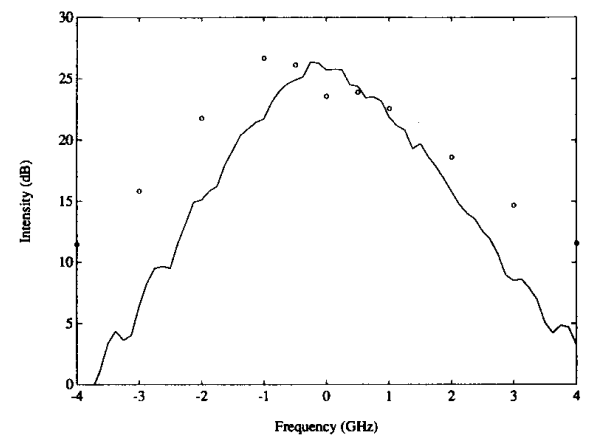

(c)

Fig. 13. Computer simulated spontaneous emission spectra (solid lines) and the peak heights of the amplified probe signal (open circles), which represent the optical response of the laser amplifier. The input optical power is of $-50 \mathrm{dBm}$ and the optical amplifier is biased at (a) 93.5 , (b) 98 , and (c) $101.5 \%$

very near threshold) the light emitted from the laser amplifier itself is not purely spontaneous but part of it is stimulated, so that this light is already partially coherent even without the injected optical signal. Therefore partially optical injection locking begins to be effective even a little below threshold. This partial injection locking effect has not been accounted for in the analytical calculation reported before [10], [25]-[27], but it is easily included in our dynamic computer simulation model. Additional information can be obtained from Fig. 15: it is that a smaller spontaneous emission rate will result in a higher sensitivity of photodetection. The mechanism be- 


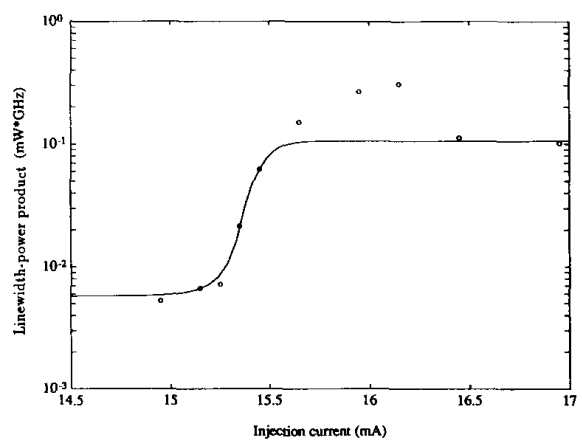

Fig. 14. Linewidth-power product calculated by the Fokker-Planck equation method (solid line) together with the computer simulated result (open points)

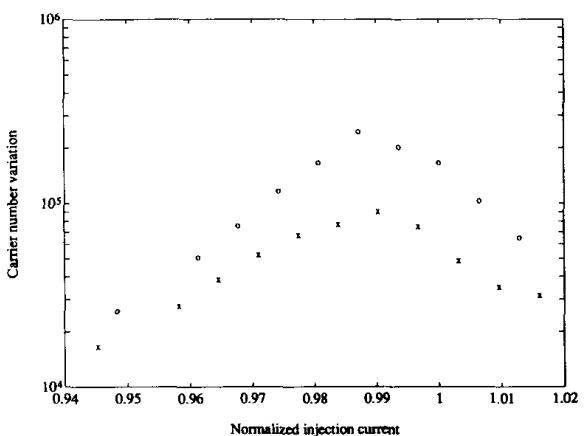

Fig. 15. Simulated carrier population variation caused by optical injection, for two values of the spontaneous emission factor $B_{* p}=2.8 \times 10^{-5}$ (open circles) and $B_{s,}=2.8 \times 10^{-4}$ (crosses).

hind this phenomenon can be explained as follows. With a higher spontaneous emission rate, the carrier population near threshold is smaller [16], thus resulting in a reduction of the photodetection sensitivity.

\section{CONCLUSION}

Linewidth of a semiconductor laser in the transition region from below to above threshold was investigated. The linewidth versus injection current characteristic was found to be nonmonotonic: a local minimum of linewidth just below threshold and a local maximum just above threshold were confirmed both experimentally and theoretically. For a semiconductor laser working below threshold as a resonant optical amplifier or optical filter, the small-signal frequency bandwidth was found to be equivalent to the spontaneous emission linewidth. The maximum photodetection sensitivity was shown to be achieved with the laser amplifier biased between $98-99 \%$ of the threshold current. Reasonable agreement between theory and experiment was obtained. A semiconductor laser with lower linewidth enhancement factor and low spontaneous emission rate was found to have a narrower optical bandwidth and higher photodetection sensitivity in narrowband optical amplification/photodetection applications.

\section{ACKNOWLEDGMENT}

The authors thank N. Caponio, P. Gambini, M. Puleo, and E. Vezzoni of CSELT for many helpful discussions and their help in the experiment. The help from P. Ansbro and $P$. Ottolenghi for the computer program are also acknowledged.

\section{REFERENCES}

[1] A. L. Schawlow and C. H. Townes," Infrared and optical masers," Phys. Rev., vol. 112, pp. 1940-1949, 1958

[2] M. Lax "Classical noise V: Noise in self-sustained oscillators," Phys. Rev., vol. 160, pp. 290-307, 1967

[3] C. H. Henry, "Phase noise in semiconductor lasers," J. Lightwave Technol., vol. LT-4, pp. 298-311, 1986.

[4] - "Theory of the linewidth of semiconductor lasers," IEEE J. Quantum Electron., vol. QE-18, pp. 259-264, 1982.

[5] R. D. Hempstead and M. Lax. "Classical noise VI: Noise in selfsustained oscillators near threshold," Phys. Rev., vol. 161, pp. 350$366,1967$.

[6] H. Risken and K. Seybold, "Linewidth of a detuned single mode laser near threshold," Phys. Lett., vol. 38A, pp. 63-64, 1972.

[7] K. Magari, H. Kawaguchi, K. Oe, and M. Fukuda, "Optical narrowband filters using optical amplification with distributed feedback," IEEE J. Quantum Electron., vol. 24, pp. 2178-2190, 1988.

[8] F. S. Choa and T. L. Koch, "Static and dynamical characteristics of narrow-band tunable resonant amplifiers as active filters and receivers," J. Lightwave Technol., vol. 9, pp. 73-83, 1991.

[9] H. Nakajima, "High-speed and high-gain optical amplifying photodetection in a semiconductor laser amplifier," Appl. Phys. Lett., vol. 54, pp. 984-986, 1989

[10] S. Kobayshi and T. Kimura, "Injection locking in AlGaAs semiconductor laser," IEEE J. Quantum Electron., vol. QE-17, pp. 681-689, 1981

[11] K. Kikuchi, "Impact of 1/f-type FM noise on coherent optical communications," Electron Lett., vol. 23, pp. 885-887, 1987; _- "Origin of residual semiconductor laser linewidth in high-power limit," Electron Lett., vol. 24, pp. 1001-1002, 1988.

[12] - " Line shape measurement of semiconductor lasers below threshold," JEEE J. Quantum Electron., vol. 24, pp. 1814-1817, 1988.

[13] K. Otsuka and S. Kobayashi, "Optical bistability and nonlinear resonance in a resonant-type semiconductor laser amplifier," Electron Lett., vol. 19, pp. 262-263, 1983.

[14] R. Hui, N. Caponio, P. Gambini, M. Puleo, and E. Vezzoni, “DFB active filter/detector for multichannel FSK optical transmission,' Electron. Lett., vol. 27, pp. 2016-2018, 1991.

[15] M. Lax, "Fluctuation and coherence phenomena in classical and quantum physics," Brandeis Univ. Summer Instit. in Theoret. Phys. vol. 2, M. Chretian, E. P. Gross, and S. Desar, Eds. New York don and Breach, 1966, p. 293.

[16] G. P. Agrawal and N. K. Dutta, Long-wavelength Semiconductor lasers. New York: Van Nostrand Reinhold, 1986, ch. 6.

[17] G. P. Agrawal and G. R. Gray. "Instensity and phase noise in mi crocavity surface-emitting semiconductor lasers," Appl. Phys. Lett. vol. 59 , pp. $399-401,1991$

[18] Z. Toffano, A. Destrez, C. Birocheau, and L. Hassine, "New linewidth enhancement determination method in semiconductor lasers based on spectrum analysis above and below threshold." Electron. Lett, vol. 28, pp. 9-11, 1992.

[19] L. G. Kazovsky, M. Stern, S. G. Menocal, Jr., and C. E. Zha, "DBR active optical filters: transfer function and noise characteristics. " $J$. Lightwave Technol., vol. LT-8, pp. 1331-1451, 1986.

[20] N. Tessler, R. N. Eisenstein, J. Salzman, U. Koren, G. Raybon, and C. A. Burrus, "Distributed Bragg reflector active optical filters," IEEE J. Quantum Electron., vol. 27, pp. 2016-2024, 1991.

[21] R. Lang " Injection locking properties of a semiconductor laser," IEEE J. Quantum Electron., vol. QE-18, pp. 976-983, 1982.

[22] M. Ajmone Marsan, S. Benedetto, E. Biglieri, V. Castellani, M. Elia, L. Lo Presti, and M. Pent, "Digital simulation of communication system with TOPSIM III," IEEE J. Selected Areas Commun. vol SAC-2, pp. 29-42, 1984.

[23] Kikuchi, "Calculated field spectra of semiconductor lasers near threshold," Electron. Lett., vol. 21, pp. 705-706, 1985. 
[24] D. Marcus, "Heterodyne detection with an injection laser-Part I: Principle of operation and conversion efficiency," IEEE J. Quantum Electron., vol. 26, pp. 85-93, 1990.

[25] T. Mukai and Y. Yamamoto, "Gain, frequency bandwidth, and saturation output power AlGaAs DH laser amplifiers," IEEE J. Quantum Electron., vol. QE-17, pp. 1028-1034, 1981.

[26] R. Hui, A. D'Ottavi, A. Mecozzi, and P. Spano, "Injection locking in distributed feedback semiconductor lasers," IEEE J. Quantum Electron., vol, 27, pp. 1688-1695, 1991.

[27] R. Hui, S. Benedetto, and I. Montrosset, "Analysis of direct-discrimination of FSK modulated optical signal using injection-locked DFB lasers," IEE Proc. J., vol. 138, pp. 276-280, 1991.

Rongqing Hui received the B.S. degree in microwave communication and the M.S. degree in lightwave technology, both from Beijing University of Posts and Telecommunications, Beijing, China, in 1982 and 1987, respec tively.

From 1982 to 1985 he taught at the department of Physics, Anhui University, Anhui, China, where his research was in optical fiber sensors. From 1985 to 1989, he was with the Department of Electronics Engineering, Beijing University of Posts and Telecommunications, where he conducted his research into coherent optical transmission systems, single-mode single-polarized optical fibers and semiconductor lasers with optical feedback. During 1989 and 1990, he held a research fellowship at the Fondazione Ugo Bordoni, Rome, Italy, where he studied optical histability, four-wave mixing and optical injection locking of semiconductor lasers. Since 1990 he has been with the Department of Electronics, Politecnico di Torino, Torino, Italy. He also held a research fellowship from the Italian Telecommunication Research Center (CSELT) during the academic year 1990-1991. $\mathrm{He}$ is currently interested in semiconductor laser devices and optical fiber communication systems.
Sergio Benedetto ( $\left.M^{\prime} 76-M^{\prime} 89-S M^{\prime} 90\right)$ received the "Laurea in Ingegneria Elettronica" (summa cum laude) from Politecnico di Torino, Italy, in 1969.

From 1970 to 1979 he was with the Istituto di Elettronica e Telecomunicazioni, first as a Research Engineer, then as an Associate Professor. In 1980, he was made a Professor in Radio Communications at the Universita' di Bari. In 1981 he was back to Politecnico di Torino as a Professor in Dat Transmission Theory at the Electronics Department. He spent 9 months during 1980-1981 at the System Science Department of the University of California, Los Angeles, as a Visiting Professor. He has coauthored two books in signal theory and probability and random variables (in Italian), and the book Digital Transmission Theory (Prentice Hall, 1987), as well as a hundred papers in leading engineering conferences and journals. He is Editor for signal design, modulation and detection for the IEEE Transactions on Communications. Active in the field of digital transmission systems since 1970 , his current interests are in the field of performance evaluation and simulation of digital communication systems, trellis coding and VLSI algorithms for telecommunications, and coherent optical communication.

Dr. Benedetto is a Senior Member of IEEE.

Ivo Montrosset was born in Aosta, Italy, on November 19, 1946. He received the laurea in electronic engineering from the Politecnico di Torino in 1971 .

From 1972 to 1986 he was with the Department of Electronics of the Politecnico di Torino, where in 1982 he became Associate Professor. From 1986 to 1989 he was Professor at the Università di Genova. Currently, he is a Professor of Optoelectronics at the Politecnico di Torino. His main activities were in the field of numerical methods for antennas and wave propagation; since 1984 he has been mainly involved in numerical simulation and design of guided-wave optics and optoelectronic devices. 